\section{Applications for Automated Particle Analysis}

\section{Robert Anderhalt and Lara Swenson EDAX Inc., Mahwah, NJ \\ bob.anderhalt@ametek.com}

\section{Introduction}

Electron microscopy and $\mathrm{x}$-ray microanalysis are common techniques in many research, development and quality labs (Garratt-Reed and Bell, 2002; Goldstein et al., 2003). As systems become ever more automated, as well as easy to use, new applications for the use of these systems develop and expand. One such application is particle analysis. These "particles" might be small loose fragments that can be applied to a conductive adhesive or a filter, embedded in epoxy and polished, or they can be inclusions within
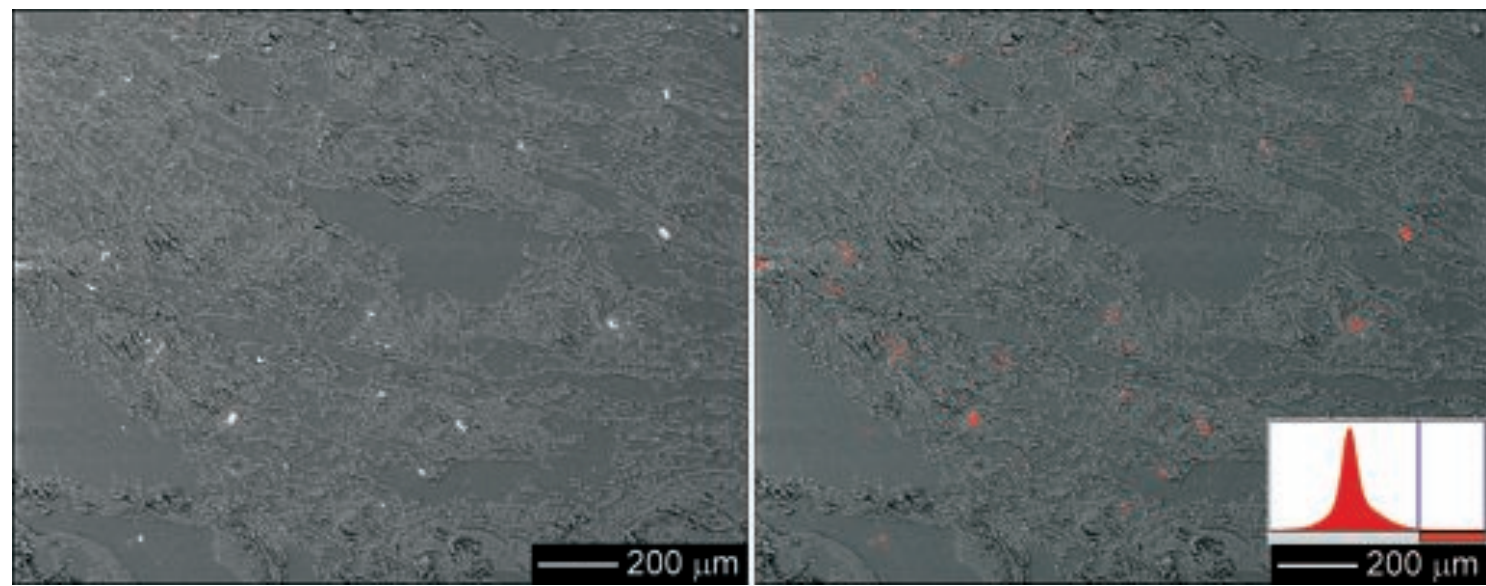

Figure 1. BSE image of lung tissue at $15 \mathrm{kV}$. The image was collected in low vacuum mode.

Figure 2. Lung tissue sample as in Fig. 1. The image histogram (inset lower right) has been thresholded to show bright particles in red. A total of 74 particles were found, EDS spectra were collected of each particle for 10 seconds and the spectra were classified with respect to a library of known phases. The total time for the data collection and analysis of this field was about 13 minutes. It would be possible to collect the spectra for a lesser time if the count rate could be increased. However, a higher count rate would require a larger spot size, higher beam currents and more damage to the sample.

\section{Requirements}

collect an EDS spectrum. The spectrum for each particle is quantified or quantitatively normalized in some way to allow the classification of the particle to a chemical phase. Lastly, after all particles are analyzed in a field, perhaps moving the stage to the location of the next field and repeating the entire process again until all fields have been analyzed. After the initial setup, all of the steps described above are done without operator intervention and all data are saved automatically. Automated particle analyses are often done as overnight and perhaps as over-the-weekend collections.

To effectively automate this process there are some necessary requirements of both the sample and of the electron microscope/ EDS hardware and software. Of the three primary requirements for automated particle analysis, the first and most important is that the particles or features of interest must be a different grayscale level as compared to the substrate or matrix. Secondly, the particles should be dispersed on the sample so that they are not touching each other and can therefore be thresholded correctly as individual particles. Lastly, the substrate or matrix will ideally be of a dissimilar chemistry from the features of interest. The second requirement is worthy of some elaboration. In image analysis software it is often possible to employ algorithms for particle separation when necessary and these another material. The analysis of particles is not without difficulty procedures can be tuned to an image as needed to determine the (Goldstein et al., 2003, p. 462-476) but with the proper attention to sample preparation and analytical procedure it is possible to analyze particles that approach the nano scale (Laskin and Cowin, 2001; Laskin et al., 2006).

Particles can be analyzed in a manual mode in which the analyst morphology of the features in the image. In automated particle analysis, data are often collected unattended, therefore the analyst is not present to judge if such a routine would be beneficial or successful. If most particles are touching, then this would indicate that the sample is not ideal for automated analysis or that the sample uses their experience and skill to detect particles, place the electron beam on the particle and to collect a spectrum by energy dispersive spectrometry (EDS). With some additional knowledge it might be possible to identify the phase of each particle and to save all the relevant image and spectral data. Manual analysis is potentially accurate but depends on the skill of the analyst and is relatively laborious and time consuming. It is possible to automate these processes yielding results that are much more consistent, thorough and efficient.

The automated analysis of particles may very well be the most powerful application of the EDS technique. When automated, this analysis combines image collection with the segmentation and the combination of pixels above or below a specified threshold into discrete particles, placing the electron beam on each particle in a spot or raster pattern to

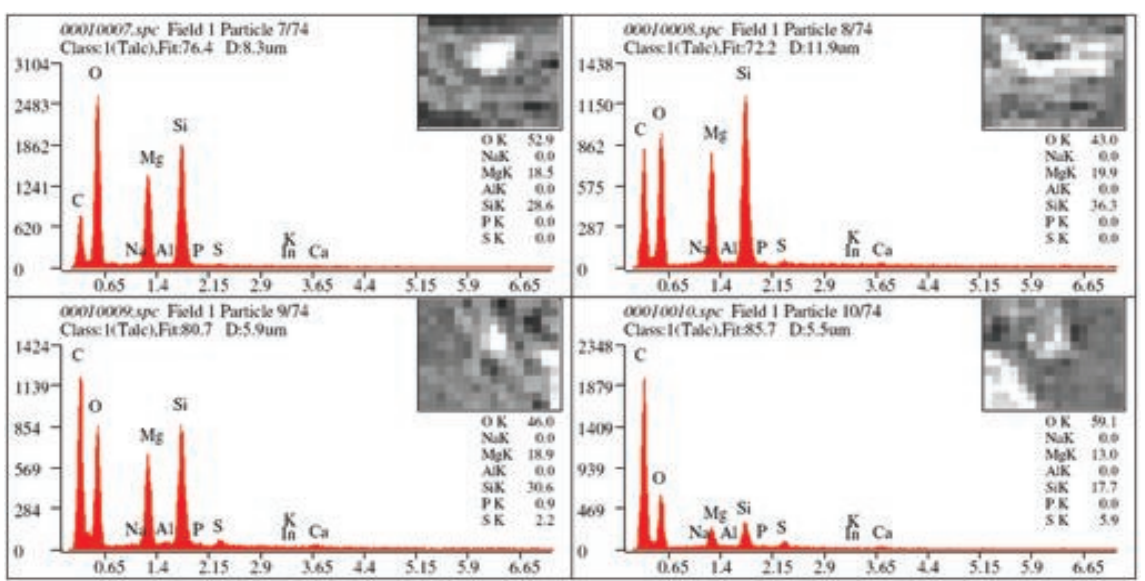

Figure 3. Particle spectra, quant results for talc ( $\mathrm{Mg}$ silicate) particles of various sizes. Some carbon is created from beam spreading into the substrate and some results from electron beam scattering above the sample surface and striking the tissue at some distance from the particle. 


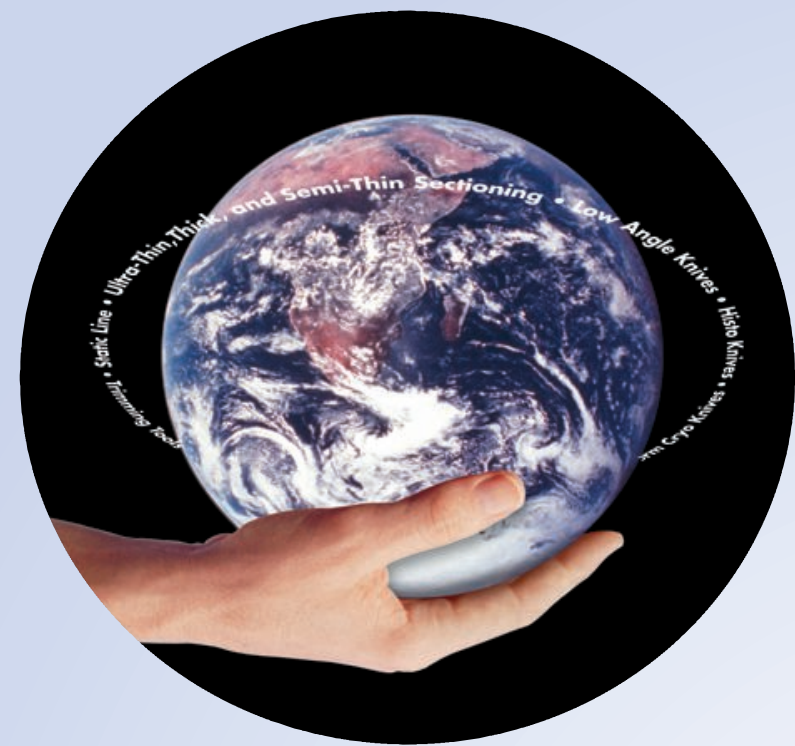

THE WORLD OF MICROSCOPY \& MICROTOMY SOLUTIONS IS WITHIN YOUR REACH WITH
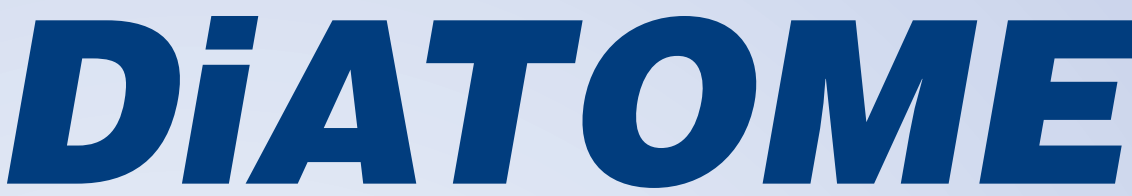

With 36 years of Developing, Innovating \& Discovering under their belts, DiATOME is the only Diamond Knife manufacturer that does not sit around waiting for technology to happen ... they make it happen leaving the other Diamond Knife manufacturers light years behind.

1970 ... DIATOME introduces the FIRST DIAMOND KNIFE.

1980 ... DIATOME introduces the FIRST CRYO DIAMOND KNIFE.

- 1983 ... DiATOME introduces the FIRST SEMI DIAMOND KNIFE FOR ALTERNATING SECTIONING.

1985 ... DiATOME introduces the FIRST LOW ANGLE $\left(35^{\circ}\right)$ DIAMOND KNIFE.

> 1986 ... DiATOME introduces the FIRST "HISTO" THICK SECTIONING DIAMOND KNIFE.

- 1989 ... DiATOME introduces the FIRST STATIC ELIMINATOR \& CRYO DIAMOND TRIMMING TOOL. (Revolutionizing Cryo Ultramicrotomy as we know it)

1999 ... DIATOME introduces the FIRST \& ONLY CRYO-P DIAMOND KNIFE. (A Cryo Knife with a Patented Platform for section pick up)

- 2001 ... DIATOME introduces the CRYO IMMUNO DIAMOND KNIFE.

(The First Cryo Knife with a Diamond Plateau: Optimized pick-up for best section quality in immuno-cytochemistry!)

\ 2004 ... DIATOME introduces the REVOLUTIONARY ULTRA SONIC DIAMOND KNIFE.

2005 ... DIATOME introduces the CRYO-TRIM $25^{\circ}$ OPTIMIZING TRIMMING WITH DIAMOND BLADES.

2006 ... DIATOME introduces the FIRST DIAMOND KNIVES FOR AFM AT ROOM \& LOW TEMPERATURES.

2006 ... DIATOME introduces the CRYO 25 DIAMOND KNIFE FOR SECTIONING FROZEN HYDRATED SPECIMENS.

\section{DIATOME THANKS THEIR CUSTOMERS FOR 36 YEARS OF PATRONAGE!}
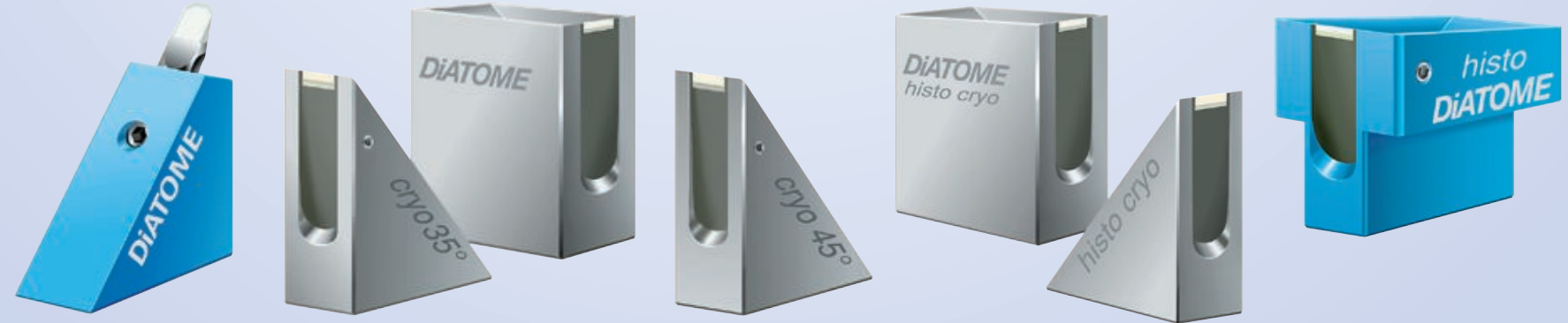

For a copy of our complete product catalog or individual brochure on any of our products, please call or write today, or visit us on the web at: www.emsdiasum.com

DIATOME - 1560 Industry Road P.0. Box 410 - Hatfield, PA 19440 tel:2 I5.4 I2.8390 fax:2 15.4 12.8450·email:sgkcck@aol.com·web:www.emsdiasum.com 


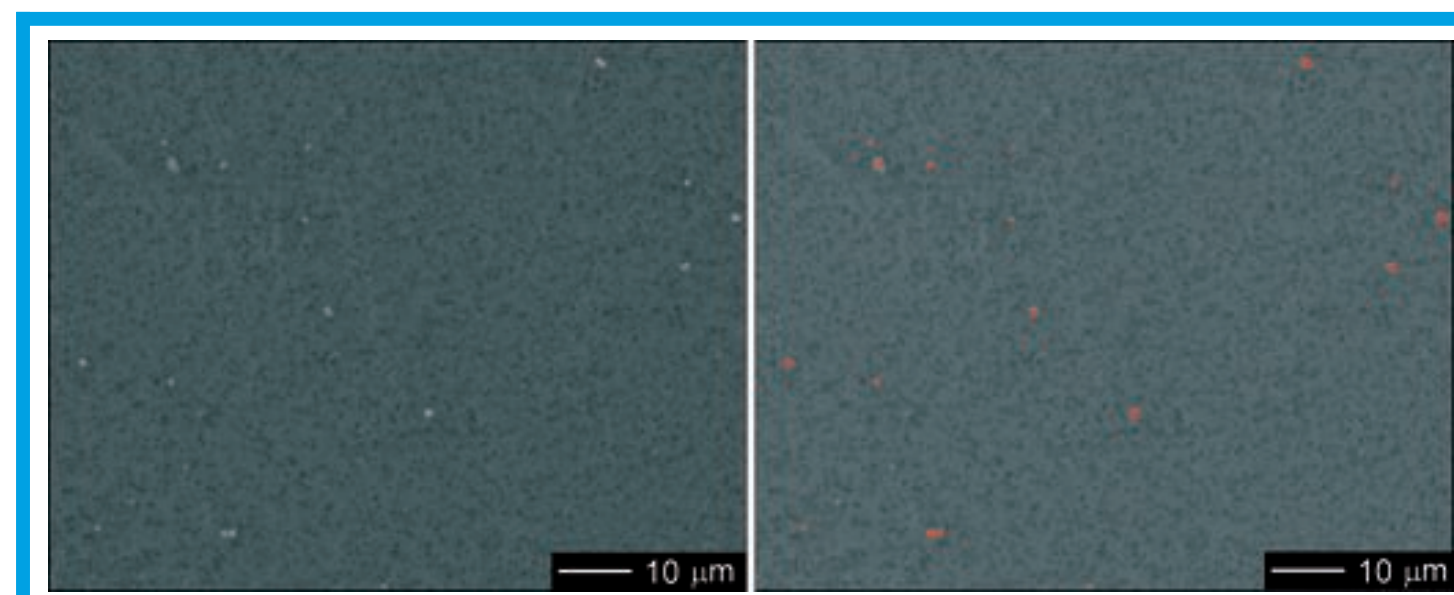

Figure 4. Particles from an air sample shown in BSE mode at a beam voltage of $15 \mathrm{kV}$. The particles are on a filter and the sample has been carbon coated. This image field is one of 9 that were collected in this dataset.

Figure 5. Air sample as in Fig. 1. The particles that are above threshold are shown in red. In all 9 fields and 153 particles were found and a spectrum was collected from each particle for 10 seconds. The total time of the analysis was 29 minutes.

preparation was not optimal. Even if a few particle aggregates are considered as a single particle, these will typically be only a small minority of the total number of particles and will not invalidate the entire dataset. The dispersion of the particles or particle loading, and the choice of substrate are setup parameters that are often under the

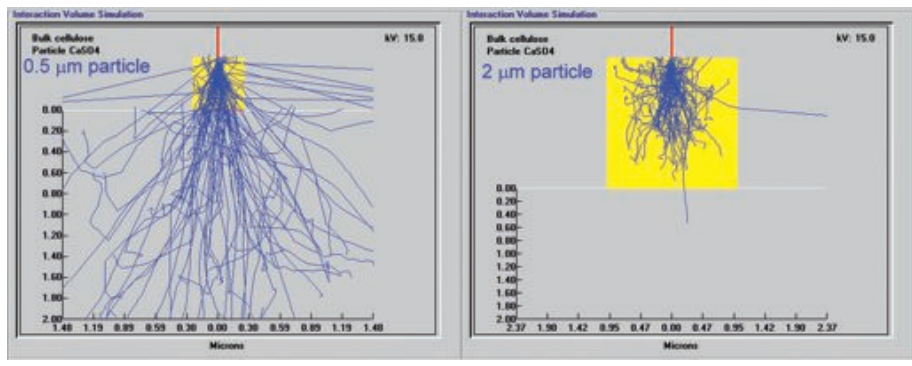

Figure 6. Monte Carlo simulations for smaller particles $(0.5 \mu \mathrm{m})$ and larger particles ( $2 \mu \mathrm{m}$ particles) at a beam energy of $15 \mathrm{keV}$ showing the penetration by the electron beam into the substrate for the smaller particles. The EDS spectra for the smaller particles would show more contribution from the substrate for these particles. If the substrate and particle have differing chemistries it is possible to ignore the major elements from the substrate.

control of the analyst and can aid the quality of the analysis.

The electron microscope is most commonly an SEM but a TEM can also be used. The ability to separate particles by graylevel from their background and from each other is typically accomplished with the use of a backscattered electron (BSE) detector. Multi-field automated analysis requires a motorized stage (at a minimum in $X$ and $Y$ ) although it may be beneficial to automate analysis of a single field for samples with high particle loading.

\section{Applications}

The application areas for automated particle analysis are large and diverse. Some of the more common or traditional applications of particle analysis include gun shot residue (GSR) and inclusions in metals. However, particle analysis techniques are also used for analysis of particles or contaminants in the hard disk industry, particles or contaminants in other devices with high-speed moving parts (gyroscopes, etc.), inclusions or constituents in aluminum alloys, inclusions in tissue, wear metals from lubricating fluids, particles in pharmaceutical products, mineral grains and inclusions in other minerals, environmental particles from air or water samples, as well as the analysis of nano-particles.
Some forms of particle analysis are almost classical in their nature. The forensic community has long been using particle analysis to locate and analyze GSR particles on a sample collected from a suspect, looking for the presence of $\mathrm{Pb}, \mathrm{Ba}$ and $\mathrm{Sb}$. Using a BSE detector, the high atomic number difference of the particles when compared to the carbon tape that the particles are collected on, allows the analysis to search for the bright particles. The steel and aluminum industries have also found particle analysis useful to locate inclusions or constituents of a variety of phases in metals. The size of the inclusions and the particle density can control mechanical properties of the alloy. In steel, the analysis will typically select inclusions that are darker than the matrix. In aluminum alloys the constituents are generally brighter than the matrix when using the BSE detector. These examples work well for particle analysis as the samples meet the necessary criteria for analysis. Several other examples are illustrated below in order to provide a sampling of areas that are applicable to automated particle analysis and to show some of variety in this field.

A paraffin-embedded section of human lung tissue was examined in low-vacuum mode using BSE at $15 \mathrm{kV}$ and a magnification of 150X (horizontal image width of $1.84 \mathrm{~mm}$; Fig. 1). Many inclusions were seen that were brighter than the graylevel of the tissue and several spectra were collected in spot mode from larger inclusions in order to confirm the feasibility of automated analysis. An automated particle analysis found 74 particles in a single field (Fig. 2) and the majority of them were classified as a magnesium silicate and presumed to be the mineral talc (Fig. 3). Because the sample was analyzed in low-vacuum mode, some $\mathrm{x}$ rays in the spectra were generated by scattered beam electrons. The scattered

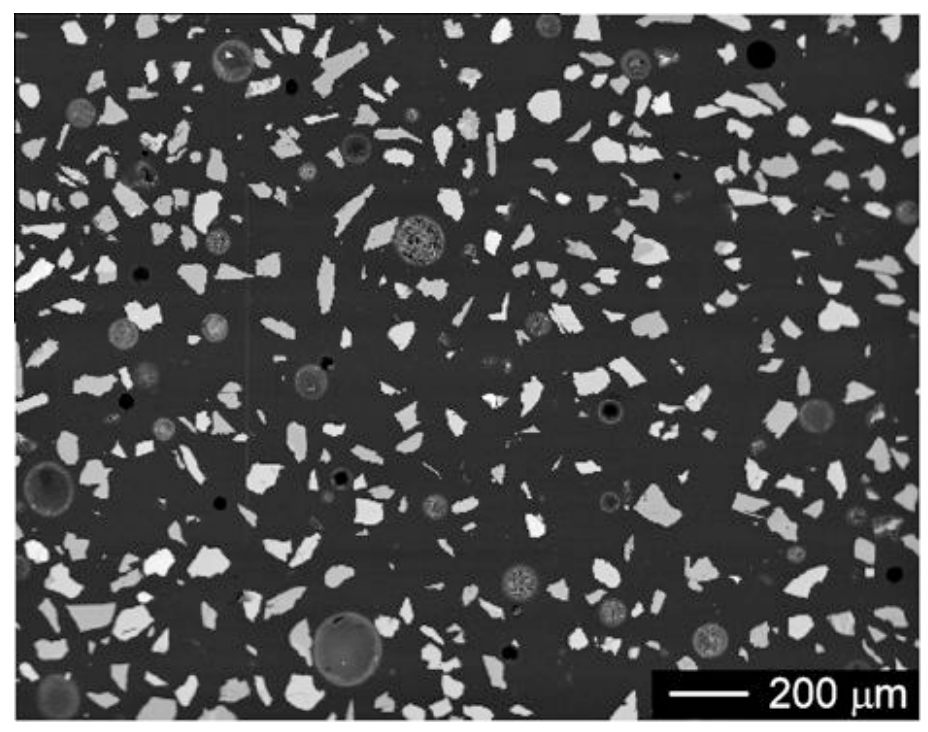

Figure 7. Particles from a mineral sample shown in BSE mode at a beam voltage of $20 \mathrm{kV}$. The particles have been embedded in epoxy, polished and carbon coated. 


\section{ENVIGION A NEW WAY OF O OMEINED}

O O MPETENCIES

\section{ZEISS NVision 40}

The new 3D CrossBeam ${ }^{\oplus}$ Workstation that combines FIB and GEMINI ${ }^{\circledR}$ SEM columns to a unique system enabling a variety of outstanding nanotech capabilities
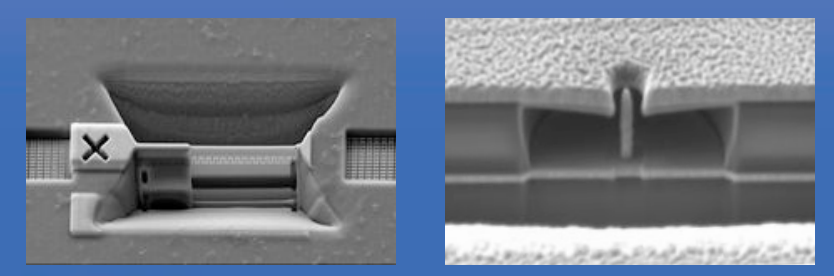


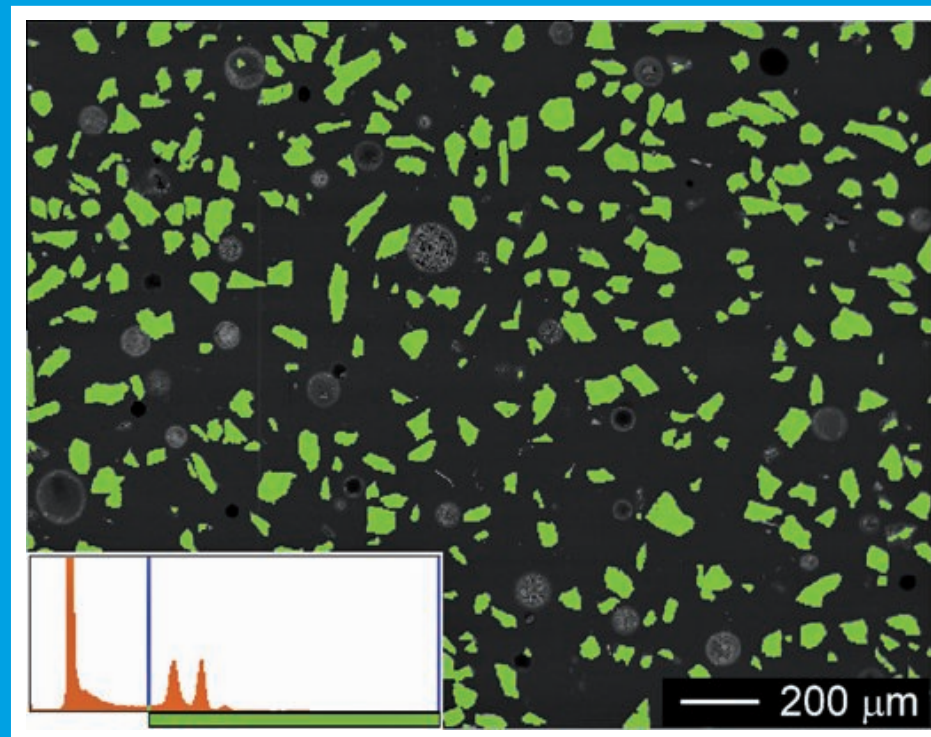

Figure 8. Mineral sample as in Fig. 1. The image histogram (inset lower left) has been thresholded to show bright particles in green. A total of 365 particles were found, EDS spectra were collected of each particle for 4 seconds and the spectra were classified with respect to a library of known phases. The total time for the data collection and analysis of this field was about 25 minutes.

electrons will primarily strike tissue and generate a spectrum dominated by carbon. This 'scattered' spectrum will combine with the spectrum from the particles generated by the unscattered beam. Fortunately, the inclusions do not have a significant carbon peak and carbon can be ignored in the analysis and is not necessary for the classification library.

Environmental particles can be sampled from an air sample onto a filter. The next sample was collected from a part of the world known to have polluted air originating from the burning of large quantities of coal. Minerals in coal are often not combusted when the coal is burned and appear in the sample in an unaltered state or they can occur in the sample in a modified composition after combustion. The sample was carbon coated and automated particle analysis was performed at a magnification of 1200X (horizontal image width of 98 micrometers) using a beam voltage of $15 \mathrm{kV}$ (Fig. 4). Spectra were collected from 153 particles found in 9 image
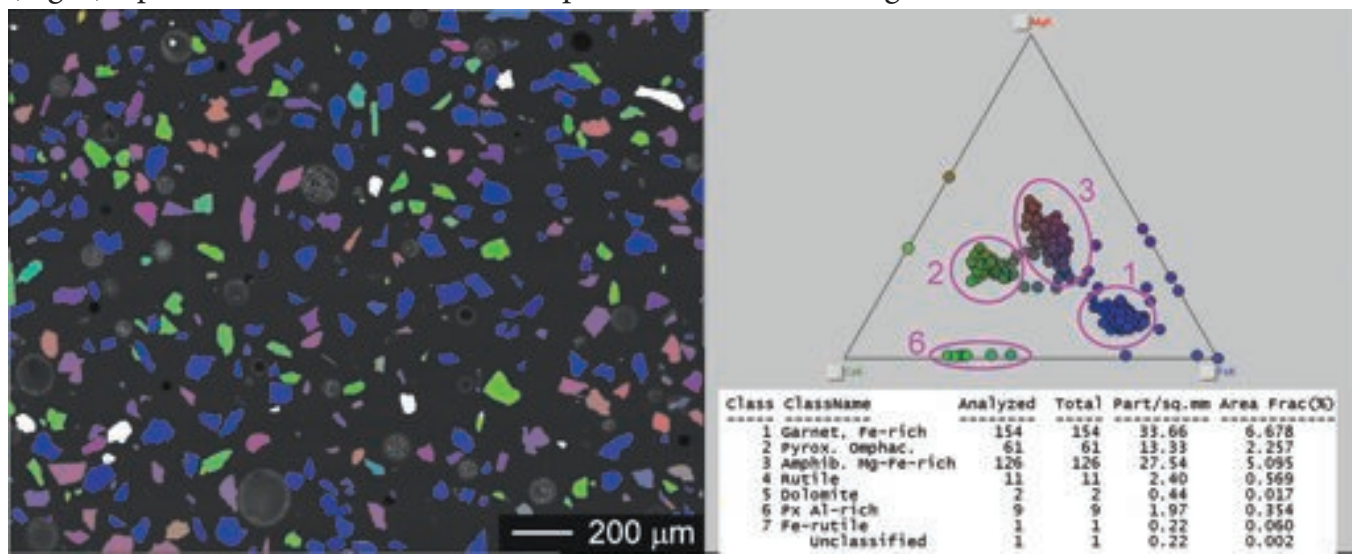

Figure 9. Ternary diagram from the mineral sample. The ternary diagram is show at right for the elements $\mathrm{Mg-Ca-Fe}$ and the mineral grains have been colorized using a red-green-blue merge. Particles with equal amounts of these 3 elements will be displayed in gray. The particles displayed in white are an indication that these particles did not contain a significant amount of these 3 elements and are really rutile (class $\# 4, \mathrm{TiO}_{2}$ or rutile contaminated with some $\mathrm{Fe}$ (class \#7). Classes 1, 2, 3 and 6 have been highlighted in the ternary diagram. Class 5 (dolomite) only contained two particles and are plotted on the line that connect the Ca and Mg apices in the ternary diagram. fields (Fig. 5). Airborne particles are typically very fine $(<3 \mathrm{mi}-$ crometers) and the minimum size used for the automated analysis was 0.19 micrometers. Particles of this size and composition will allow $15 \mathrm{keV}$ energy electrons to pass through the particle into the substrate/filter (Fig. 6). The substrate contribution to the spectrum will be significant for the smallest particles and consists primarily of carbon. In this analysis it is also possible to ignore the carbon peak and concentrate on the other elements to characterize even the smallest particles because carbon is not present in the particles of interest, or it is not needed in order to make a useful classification by chemistry.

A final example involves crushed rock particles from a mining operation. The purpose of the crushing is to liberate specific ore mineral(s) so that they might be concentrated with a minimum of contamination from other phases. In the example shown in Fig. 7 the mineral of interest is rutile $\left(\mathrm{TiO}_{2}\right)$. The mineral sample was embedded in epoxy, polished, and then carbon coated. The sample was analyzed using a beam voltage of $20 \mathrm{keV}$ at a relatively low magnification (50X; horizontal image width of $2.46 \mathrm{~mm}$ ). Most of the 365 grains are between 20 and 100 micrometers in diameter (Fig. 8). All mineral grains have been analyzed in this example and the ternary diagram shows the ability to resolve and pseudo-color the phases by composition using a red-green-blue merge on $\mathrm{Mg}$ Ca-Fe respectively (Fig. 9). The result of the analysis is summarized by the number of particles in each classification (see inset in Fig. 9) while a spreadsheet is created that contains all of the particle morphometric parameters (area, size, shape, etc.), the chemistry data and the result and fit of the classification.

\section{Conclusions}

Automated particle analysis is a viable approach for a wide range of sample types and applications. The primary requirements are that the images of the sample should have gray level contrast that allows the separation of particles or features of interest from the remainder of the sample and that the particles can be separated from each other. Particles can be located, sized, analyzed by EDS and sorted into phases. Automated particle analysis has many advantages over a manual analysis of particles including speed, thoroughness, consistency and an extensive digital record of the data. 


\section{Let 4pi take you beyond the capability of any SEM/STEM on the market with}

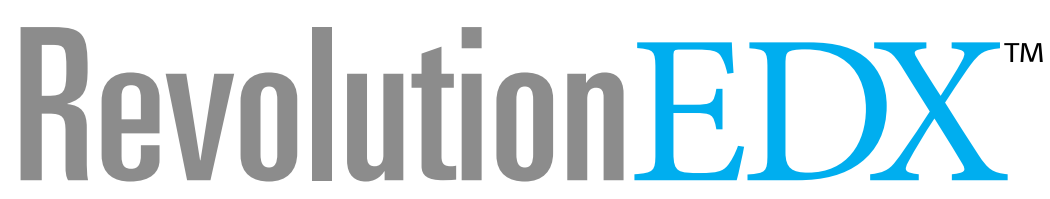

\section{X-ray Microanalysis Systems}

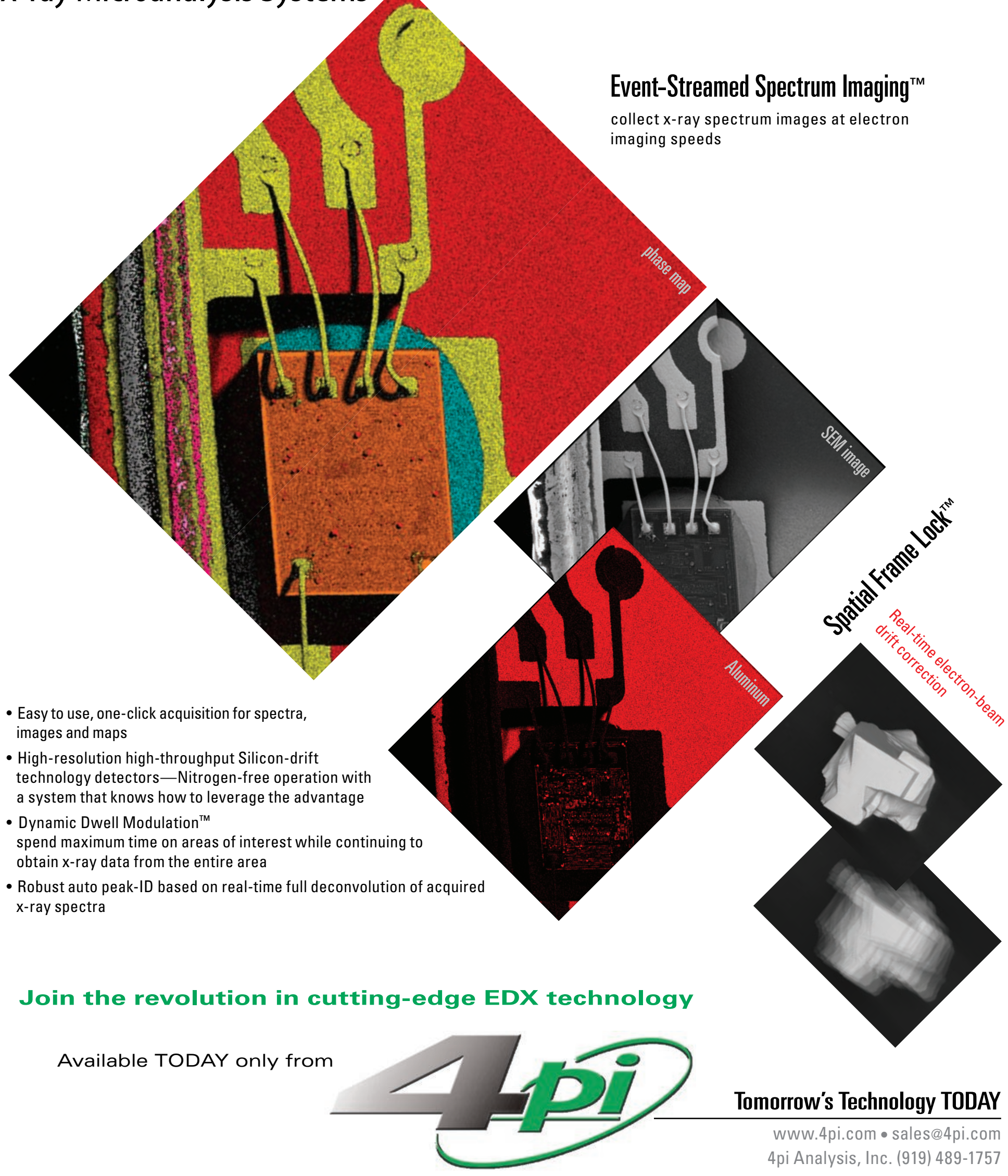

